\title{
A rare presentation of melanoma as a retroperitoneal mass: A case report and a brief review of the literature
}

\author{
Arvind Randhawa ${ }^{1}$, Niket Sonpal ${ }^{2}$, Stephen Machnicki ${ }^{3}$, Colette Spaccavento ${ }^{1}$, Amory Novoselac $^{1}$ \\ 1. Department of Hematology-Oncology, Lenox Hill Hospital, New York - North Shore Long Island J ewish Health System, \\ USA. 2. Department of Medicine, Lenox Hill Hospital, New York-North Shore Long Island Jewish Health System, USA. 3. \\ Department of Radiology, Lenox Hill Hospital, New York - North Shore Long Island J ewish Health System, USA
}

Correspondence: Arvind Randhawa. Address: Department of Hematology-Oncology, Lenox Hill Hospital, New York -North Shore Long Island J ewish Health System, USA. E-mail: arandhawa@nshs.edu

Received: November 8, $2012 \quad$ Accepted: December 7, 2012

Online Published: January 9, 2013

DOI : $10.5430 /$ jst.v3n2p10

URL: http://dx.doi.org/10.5430/jst.v3n2p10

\section{Abstract}

Malignant melanoma presenting as a retroperitoneal mass is a rare manifestation of this disease. Melanoma, a malignant tumor of melanocytes, is a form of skin cancer with about 160,000 new cases of melanoma yearly worldwide. More than 53,000 people in the United States are diagnosed with melanoma every year. We present a rare case of malignant melanoma presenting as a retroperitoneal mass.

\section{Key words}

Retroperitoneal mass, Malignant melanoma

\section{Case report}

A 77 year old African American male presented with fatigue, abdominal pain, decreased appetite and 40 lbs weight loss over the period of 2-3 months. The patient had a CT scan of the abdomen and pelvis done as an outpatient which demonstrated a large heterogeneous, lobulated mass in the sub hepatic space measuring approximately $16.7 \mathrm{~cm} \times 11 \mathrm{~cm} \times$ $13 \mathrm{~cm}$ (Figure 1). This was thought to be a retroperitoneal mass. He was noted to have new onset anemia with positive stool hem occult. The patient was admitted to the hospital for further evaluation. His past medical history was remarkable for hyper lipidemia and benign prostatic hypertrophy. He is a former smoker and denied alcohol or illicit drug use. The patient was noted to be a cachetic, frail elderly man. Cardiopulmonary evaluation was unremarkable. Abdomen was soft with tenderness and fullness in the right upper quadrant, bowel sounds were present. Skin exam did not reveal any lesions. His white blood cell count was $6.5 \times 10^{3}$; Hemoglobin 9.6 and platelet count $451 \mathrm{~K}$. His basic chemistry panel was within normal limits. The patient received two units of packed red blood cells with appropriate response. He underwent an endoscopic evaluation for the GI bleed. Endoscopy revealed a mass infiltrating the duodenum. This infiltration of the mass was not appreciated on the previous CT scan. The patient underwent a PET/CT. The PET/CT again showed a large heterogeneous, lobulated sub hepatic mass. The peripheral aspect of the mass had intense FDG activity. Central portions of the mass were not FDG-avid, suggesting that they were necrotic. Two small hyper metabolic foci were seen in the liver, consistent with metastases. 
Figure 1. CT scan abdomen/pelvis Axial and coronal images from a CT scan show a large heterogeneous mass in the right upper quadrant. The mass has areas of low density that likely represent necrosis. The mass is contiguous with the inferior aspect of the liver, the duodenum, the right adrenal gland, and the pancreatic head.
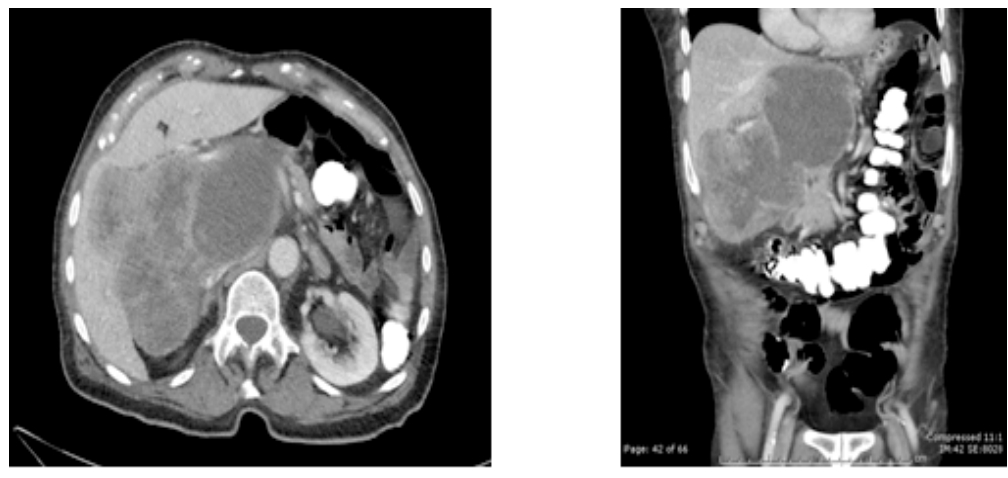

The patient underwent CT guided fine needle aspiration of the mass. The cytology from the aspiration was consistent with metastatic malignant melanoma. Immuno histochemical stains performed on the cell block demonstrated positive staining for vimentin, HMB45, melan-A and focally for S100 (Figure 2 and Figure 3). An extensive skin exam was performed and no skin lesions were identified. The patient denied any history of skin lesions being excised in the past or any family history of melanoma. The patient was not a candidate for aggressive therapy due to poor and declining performance status.

Figure 2. H\&E stains: Cohesive groups of large cells with pleomorphic nucleoli and pigmented cells.
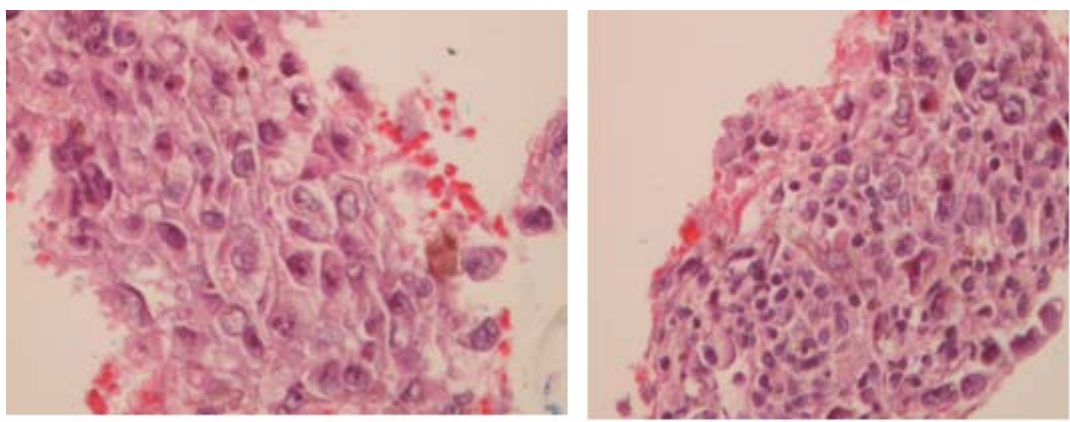

Figure 3.

Immunohistochemical stains: shows negativity for pan keratin but positivity for HMB45 and Melan A.

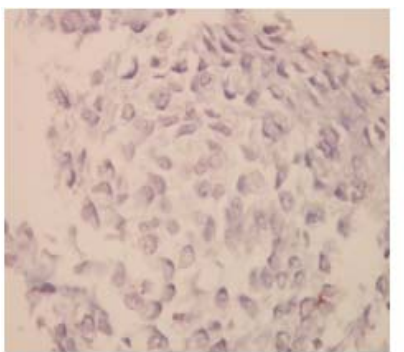

Pan Keratin

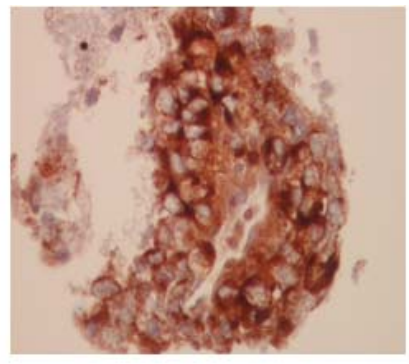

HMB45

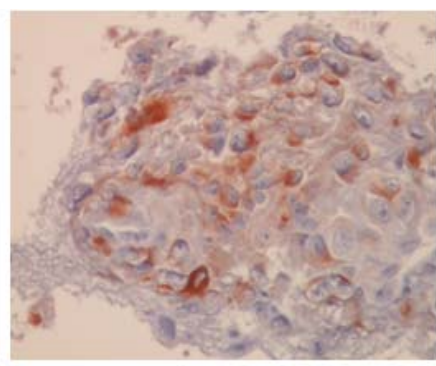

Melan-A

\section{Discussion}

Melanoma is known to have varied presentations and can present in any organ, but a retroperitoneal presentation is unusual as evidenced by a few reports in the literature. Melanoma is a neoplastic disorder produced by malignant transformation of the normal melanocyte. Melanocytes are cells responsible for the production of the pigment melanin. 
Malignant melanoma is the sixth-most-common U.S. cancer diagnosis. It affects all age groups and the median age at diagnosis is 50 years. When melanoma arises in the skin, it usually arises from melanocytes at the dermal epidermal junction. In addition, there are alternate presentations, including mucosal melanomas, ocular melanomas, metastatic melanomas from unknown primary sites, and presumed primary visceral melanomas. In the National Cancer Database (NCDB), $91.2 \%$ of melanomas are cutaneous, $5.3 \%$ are ocular, $1.3 \%$ are mucosal, and 2.2\% are of unknown primary site. Risk factors for melanoma include sun exposure, cutaneous phenotype, and precursor skin lesions (i.e. dysplastic nevi, benign nevi).

Early detection and recognition of melanoma is the key to improving overall survival of patients with malignant disease. The signs of early melanoma are based on the clinical appearance of the pigmented lesions in addition to any change in the existing mole. A history of recent change in the pigmented lesion or a new pigmented lesion requires a biopsy. The proper biopsy is essential not only to establish a diagnosis but also for prognosis. Change in size, color and border are important clues to the diagnosis of melanoma. Although melanomas are most frequent in sun-exposed areas of the skin, these tumors may arise at a number of other sites (e.g., nail bed, mucosal surfaces, and eye).

Cutaneous and noncutaneous melanomas differ in their epidemiology, even though they have a shared cell of origin. Noncutaneous melanomas tend to present at an older age and to be diagnosed at a more advanced stage. Melanomas at these unusual sites generally carry a worse prognosis than those arising in the common cutaneous locations. Non cutaneous melanomas include: acral, subungual, mucosal (anorectal, head and neck, respiratory, alimentary and genitourinary tract). Here the patients typically present with bleeding, a mass, pain, or a change in bowel habits. Whenever melanoma is found in the gastrointestinal tract or in retroperitoneal site, a primary cutaneous lesion is nearly always documented. It is rarely absent, as in this case. Our patient did not have history of melanoma or excised skin lesions. This patient presented with a huge retroperitoneal mass and nonspecific gastrointestinal symptoms not suggestive of a primary retroperitoneal tumor. Primary retroperitoneal tumors are rare and most often they are sarcomas or tumors arising from adrenal glands. Our patient's case was initially suspicious for sarcoma based on the CT scan findings. Immuno histochemical studies led to the correct diagnosis in our patient. Histopathological and immunohistochemical analyses are indispensable to the diagnosis of melanoma. Extra-cutaneous melanomas exhibit the same immune histochemical and ultra-structural features as their cutaneous counterparts. S-100 protein, HMB-45, melanin A, vimentin are used as immunohistochemical tools in the diagnosis of melanoma. In our patient, immunohistochemical stains show positivity for HMB45 and Melan-A findings that were conclusive for the diagnosis of malignant melanoma.

In conclusion, melanoma has varied presentations and to our knowledge this is a very uncommon presentation of malignant melanoma. This case is highlighted for its unusual presentation and therefore melanoma should be part of the differential diagnosis of a retroperitoneal mass.

\section{References}

[1] Geller AC, Miller DR, Annas GD, et al. Melanoma incidence and mortality among US whites, 1969-1999. JAMA. $2002 ; 288: 1719$. PMid:12365954 http://dx.doi.org/10.1001/jama.288.14.1719

[2] Breslow A. Thickness, cross-sectional areas and depth of invasion in the prognosis of cutaneous melanoma. Ann Surg. 1970; 172: 902. PMid:5477666 http://dx.doi.org/10.1097/00000658-197011000-00017

[3] Paek SC, Sober AJ, Tsao H, et al. Cutaneous melanoma. In: Fitzpatrick's Dermatology in General Medicine, 7th, Wolff K, Goldsmith LA, Katz SI, et al. (Eds), McGraw Hill Medical, New York. 2008; I: 1134.

[4] Hussein MR; Extracutaneous malignant melanoma. Cancer Investigation: 2008 Jun; 26(5): 516-534. PMid:18568775 http://dx.doi.org/10.1080/07357900701781762

[5] Liu et al. Primary retroperitoneal malignant melanoma: a case report. Oncology Letter. 2011 Nov; 2(6): $1107-1111$.

[6] Mahkmari et al. Primary retroperitoneal malignant melanoma. Pan African Medical Journal. 2012 June; $12: 20$. 